\title{
ALEEDSA: Augmented Reality for Interactive Machine Learning
}

\section{Conference Paper}

Author(s):

Ferrario, Andrea (D); Weibel, Raphael (D); Feuerriegel, Stefan

Publication date:

2020-04

Permanent link:

https://doi.org/10.3929/ethz-b-000440530

Rights / license:

In Copyright - Non-Commercial Use Permitted

Originally published in:

https://doi.org/10.1145/3334480.3382937

\section{Funding acknowledgement:}

183149 - The digital manager: How digitalization ist transforming managerial decision-making (SNF) 


\section{ALEEDSA: Augmented Reality for Interactive Machine Learning}

Andrea Ferrario ETH Zurich

Zurich, Switzerland

aferrario@ethz.ch

Raphael Weibel

ETH Zurich

Zurich, Switzerland

rweibel@ethz.ch

Stefan Feuerriegel

ETH Zurich

Zurich, Switzerland

sfeuerriegel@ethz.ch

\begin{abstract}
In this work, we present ALEEDSA: the first system for performing interactive machine learning with augmented reality. The system is characterized by the following three distinctive features: First, immersion is used for visualizing machine learning models in terms of their outcomes. The outcomes can then be compared against domain knowledge (e.g., via counterfactual explanations) so that users can better understand the behavior of machine learning models. Second, interactivity with augmented reality along the complete machine learning pipeline fosters rapid modeling Third, collaboration enables a multi-user setting, wherein machine learning engineers and domain experts can jointly discuss the behavior of machine learning models. The effectiveness of our proof-of-concept is demonstrated in an experimental study involving both students and business professionals. Altogether, ALEEDSA provides a more straightforward utilization of machine learning in organizational and educational practice.
\end{abstract}

\section{Author Keywords}

Augmented reality; Mixed reality; Collaborative interaction; Machine learning

\section{CCS Concepts}

•Human-centered computing $\rightarrow$ Mixed / augmented reality; $\cdot$ Computing methodologies $\rightarrow$ Machine learning; 


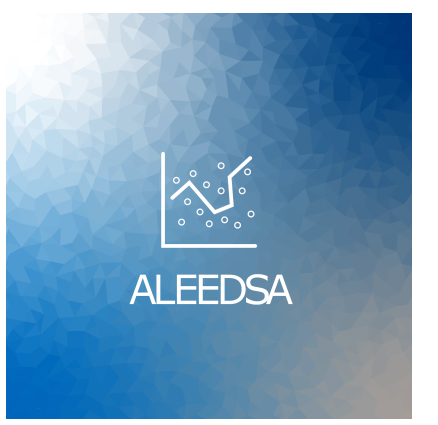

Figure 1: ALEEDSA: Logo. ALEEDSA is pronounced as "Ah-lee-zah", from the Hebrew name "Aliza", which means "joyful". It is short for Augmented LEarning Experience Data Science App.

\section{Introduction}

Machine learning (ML) provides systems that learn to perform task without explicit instructions [12]. Recently, ML has found widespread traction in businesses and organizations, where it helps in automating tasks that are perceived as tedious, repetitive, bias-prone, or that are constrained to domain experts. Real-world examples of ML include crime prediction, sales forecasts, personalized treatment planning, and language translation (e.g., Google Translate).

Despite the potential for value creation, adoption of $M L$ in practice faces several challenges [16]. These challenges originate foremost from the fact that $\mathrm{ML}$ is built upon probabilistic algorithms [12], that is, algorithms make inferences under uncertainty. However, practitioners strive to understand how ML models arrive at prediction outcomes, so that they can eventually trust the model $[10,14,19]$. Hence, actual use cases in practice demand that several questions regarding a given $\mathrm{ML}$ model be answered before deployment, such as the following: What is the performance of a ML model? Where is ML making errors? How is ML handling rare events? Why does the ML model output a certain prediction for a given data point? How do ML model predictions compare against domain knowledge?

In order to address the above challenges, we argue that a viable solution is offered by augmented reality (AR) [1]. Augmented reality provides embodied cognition, whereby digital content is superimposed on physical reality, as well as new ways of interacting with information in a multimodal, immersive, and collaborative context [3, 13]. Immersion allows for the visualization of complex relationships, so that users grasp the logical relationships between them intuitively [17]. For example, it has been argued that the use of augmented reality can support users' memory skills [6], and provides a basis for immersive, remote experiences [9].
Augmented reality is especially effective in use cases that involve complex visualization or require frequent interactions. Examples include the domains of engineering [18] medicine [4], and education [15]. Yet, to the best of our knowledge, augmented reality has not been used for directly interacting with $\mathrm{ML}$ model outcomes. In the status quo, outcomes of ML models are interpreted by practitioners via on-screen visualization, often only a two dimensional $[2,11,14]$. There have been a few attempts to extend these visualizations via interactive elements [8, 7], and yet the potential of AR has remained untapped.

In this paper, we introduce ALEEDSA (see Fig. 1), the first system for performing interactive machine learning with augmented reality. The system addresses the above mentioned challenges in $\mathrm{ML}$ by supporting users in designing, evaluating, and interpreting ML models via AR. For this, our proof-of-concept is built on top of Unity and Microsoft HoloLens, which allows us to incorporate the following three features: (1) Immersion ensures a graphically rich representation of prediction outcomes. Based on it, users can obtain counterfactual explanations and compare prediction outcomes against domain knowledge. (2) Interactivity facilitates an engaging yet intuitive use, even in the presence of high-dimensional data. This should allow for rapid modeling. (3) Collaboration is integrated via a shared workspace. Here users can interact with the same ML model. The effectiveness of our proof-of-concept is evaluated based on a user experience questionnaire. For this, we conducted an experiment with $N=21$ subjects from two different backgrounds (i. e., students and working professionals).

\section{Concept}

The rationale behind ALEEDSA is that users in the field of machine learning benefit from the use of augmented reality. Specifically, augmented reality should improve the user ex- 
perience along three dimensions - immersion, interactivity, and collaboration - as detailed in the following.

\begin{tabular}{|l}
\multicolumn{1}{c|}{ Activity } \\
\hline $\begin{array}{l}\text { ML model I: } \\
\text { training }\end{array}$ \\
$\begin{array}{l}\text { ML model I: } \\
\text { outcomes } \\
\text { interpretation }\end{array}$ \\
feedback \\
$\begin{array}{l}\text { ML model II: } \\
\text { training }\end{array}$ \\
ML model II: \\
outcomes \\
interpretation
\end{tabular}

Figure 2: lllustrative example of user activities in ALEEDSA. It shows how users train, evaluate, and explain prediction models iteratively using both stages of ALEEDSA's interface. Feedback is provided at the end of each activity.
Immersion is achieved by designing the user interface such that it naturally aids users in understanding how ML models arrive at prediction outcomes. Thereby, users should be able to answer questions such as, e. g.: What is the performance of a ML model? Where is ML making errors? Are possible patterns of discrimination emerging? How is $\mathrm{ML}$ handling rare events? Why does the ML model output a certain prediction for a given individual? Based on the aforementioned questions, users can compare predictions from different $M L$ models and, specifically, to domain knowledge. In addition, augmented reality allows for an interactive, three dimensional visualization. As opposed to $2 \mathrm{D}$, it displays an additional predictor, thereby making highdimensional relationships easier to identify.

Interactivity spans the complete ML pipeline, namely (1) designing the ML model, including the model choice and hyperparameter tuning; (2) evaluating the overall prediction performance; and (3) interpreting prediction outcomes via post hoc visualization. For instance, interactivity renders it possible for users to quickly update parameter configurations, so that they can assess the effect on the prediction outcomes. Here the rationale is that interactivity makes such analyses straightforward and, in particular, rapid.

Collaboration is needed in machine learning so that ML engineers and domain experts can co-develop models.

This is relevant in practice, especially in domains (such as healthcare) that involve high-stakes decisions. Here ML engineers receive instructions from line managers. Then they must design models accordingly, and, eventually, communicate the results to end-users so that the trained model can be compared to domain knowledge. For this reason, ALEEDSA supports collaboration by design: users have ac- cess to a shared workspace where they can develop ML models jointly by interacting with the same visualizations at the same time.

\section{ALEEDSA}

ALEEDSA uses augmented reality for interactive machine learning. It covers the complete ML pipeline, that is, it should allow users to design, evaluate, and interpret ML models. Thereby, ALEEDSA helps users better understand how ML models are built and their predictions are inferred in a realistic setting. To this end, it is built upon (1) immersion in the interface design, (2) interactivity, and (3) collaboration techniques.

To illustrate how ALEEDSA could be used in practice, we provide an example in the following. Let us consider a realworld use case in which a ML engineer and a line manager collaborate in developing a ML classifier for predicting the creditworthiness of customers. An exemplary activity diagram is depicted in Fig. 2, illustrating the iterative cycle of designing, evaluating, and interpreting different ML classifiers.

Interfaces

In ALEEDSA, the interface comprises two components - a prediction explorer and a model designer - as follows.

The prediction explorer is responsible for visualizing the outcomes of a ML model across different predictors. It is based on a three-dimensional scatter plot; see Fig. 3 (top). Each axis corresponds to one predictor, with labels denoting its name. Spheres represent individual data points that are colored according to the prediction outcome. In addition, a side menu is displayed next to the diagram and contains context-sensitive information; see Fig. 3 (bottom). It comprises the ID of a data point, the prediction outcome, and the complete list of values from all predictors. A sep- 
arate menu is available that lists the overall performance scores of the model.

The model designer provides a three-dimensional interface for specifying the parameters of a ML model (see

Fig. 5). In a collaborative setting, this menu is available to all users, so that the specification can occur collaboratively. To this end, users proceed by selecting (1) the model class (e. g., generalized linear models or neural networks), (2) the variables to be used for training, and (3) the hyperparameters corresponding to a model. Afterwards, the predictions from the desired ML model are retrieved and the outcomes are sent to the prediction explorer for display.

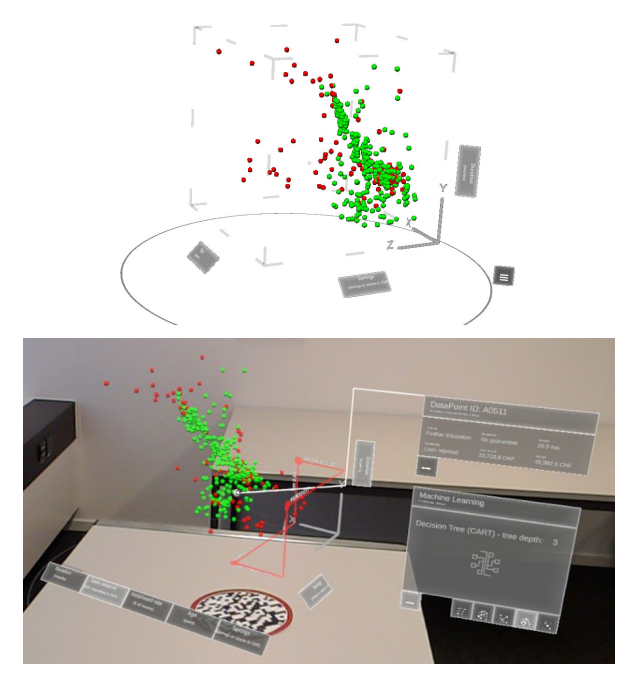

Figure 3: Prediction explorer in ALEEDSA. Top: 3D scatterplot as visualization of prediction outcomes. Bottom: additional menu of the prediction explorer that appears when a data point is selected in order to show context-sensitive information.

\section{Interactivity}

Both components - prediction explorer and model designer - are interactive. Depending on the view of users, the corresponding elements are highlighted and can be selected via a gesture (i. e., air tap). The corresponding actions vary according to the component.

In the prediction explorer, a user can focus her view on a single data point in order to display the values of that data point on the $(x, y, z)$ axes. To facilitate a better orientation in $3 \mathrm{D}$, additional ticks on each axis are added. If the user's view focuses on a single data point (i. e., highlighted by a lighter color), then the air tap gesture opens the side menu with further details concerning the data point, as detailed above. In addition, all axes are designed as buttons that change which predictors are depicted.

The prediction explorer further supports the interpretation of ML outcomes via counterfactual explanations [5]. These provide answers to questions such as, e.g., "Which predictor must be changed to obtain a different prediction?".

Following the previous example, this provides pragmatic explanations as to why a loan applicant was not predicted to be creditworthy. To do this, additional "synthetic" data points are shown (see Fig. 6; gray point). When such a data point is selected by air tapping, the counterfactual explanation is displayed in the context-sensitive side menu.

In the model designer, all displayed elements are designed as interactive buttons based on which a parameter configuration for the ML model can be selected.

\section{Collaboration}

ALEEDSA enables collaboration by providing two workspaces, namely a private and a shared workspace (see Fig. 4). In the private workspace, users can analyze ML outcomes independently and also interact with line managers using the 


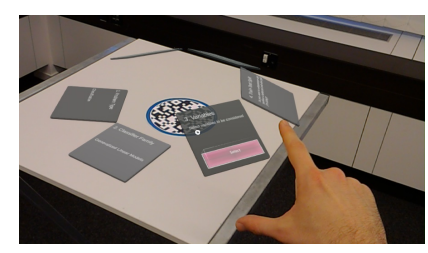

Figure 5: Model designer in ALEEDSA. Users interact with the augmented reality elements to select the model class, the variables to be used in training and to specify the hyperparameters.

Training of the ML model is

triggered by air tapping a dedicated augmented reality button. web interface. The shared workspace is reserved for collaboration among multiple ALEEDSA users, in which they can jointly discuss and interact in real time while having access to the same visualization. This allows for different collaborative activities: (1) sharing insights on ML model outcomes previously analyzed in a personal workspace; (2) training ML models; and (3) investigating about outcomes of ML models with counterfactual explanations and submitting results to line managers.

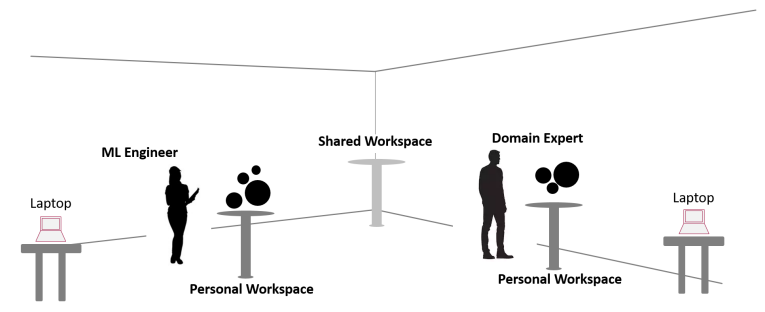

Figure 4: Collaboration in ALEEDSA: each user has a persona workspace and a laptop (e.g. to receive tasks and feedback from line managers). A shared workspace enables collaboration. This setup enables settings in practice, where $M L$ engineers have to collaborate with domain experts and augmented reality is used together with other traditional systems.

\section{Implementation}

The implementation of ALEEDSA is based on a model-viewcontroller design pattern. The controller represents the back-end. It implements the logic when the ML parameters are updated or when counterfactual explanations need to be computed. The controller operates on the current state, that is, the model comprising both the dataset and the $\mathrm{ML}$ parameters. The view for a single user is given by two different interfaces: one user interface is the augmented reality device with three dimensional visualizations. For reasons of usability, a secondary user interface was developed in the form of a web interface (e.g., to load a dataset). We reiterate that, due to collaboration, ALEEDSA comprises multiple such interfaces.

In our proof-of-concept, augmented reality is developed for Microsoft HoloLens 1. ${ }^{1}$ The HoloLens provides a headmounted display where augmented reality is superimposed on top of the regular view and, for that reason, its is beneficial in multi-user collaborations. The HoloLens was programmed using the Unity engine as shipped by the Windows 10 SDK. ${ }^{2}$. The back-end (i. e., the controller) was developed as an Azure application that communicates with both HoloLens and web interface via asynchronous notifications (REST messages) using the SignalR library. It also ensures that, in multi-user settings, the interfaces are synchronized.

\section{Early User Feedback}

In order to collect early user feedback, we performed an experimental evaluation based on a prototype of ALEEDSA. The objective was two fold: First, the experiment should validate our working hypothesis, namely that augmented reality is effective for machine learning. Second, we wanted to collect user feedback in order to support future development.

For the proof-of-concept evaluation, ALEEDSA was deployed with a simple case study, in which users were supposed to model credit risk at a fictitious bank. Specifically, users had to develop a machine learning model that predicted creditworthiness for loans based on several customer variables (e. g., loan amount, duration, customer age). To mimic reality, the case study was designed as a role play, in which

\footnotetext{
${ }^{1}$ https://docs.microsoft.com/en-us/hololens/

hololens1-hardware

${ }^{2}$ https://unity.com/
} 

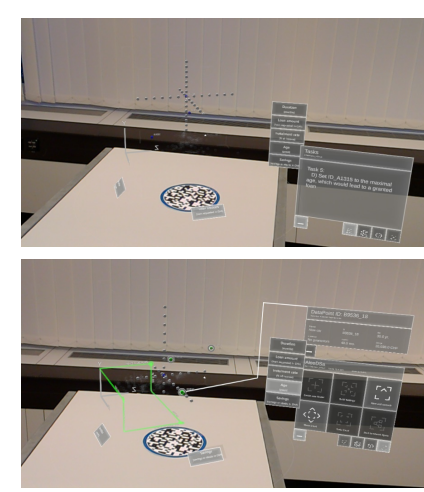

Figure 6: Counterfactual explanations inside the prediction explorer. Top: starting visualization of "synthetic" data points in the "prediction explorer", for a selected outcome (in blue). The "synthetic" data points stretch out along the three axes, by definition of counterfactual explanation. Bottom: three "synthetic" data points being selected for three ML model outcomes. A "Save and Commit" button allows users to share results with line managers. users acted as a ML engineer who had to communicate with a risk officer as a domain expert. Here users had to inspect the performance of a given ML model.

The prototype was tested based on 21 subjects ( 6 female) who represent different user groups from educational and organizational settings: On the one hand, 13 subjects were college students pursuing a master's degree in management, technology, and economics (average age: 25). On the other hand, 8 subjects were working professionals (average age: 36 ). IRB approval was obtained from ETH Zurich.

Overall, $67 \%$ of the subjects reported that interactive visualizations in augmented reality enabled them to better understand data. Similarly, $52 \%$ of the subjects confirmed that ALEEDSA helped them more quickly infer relationships in the data. Moreover, the majority of subjects ( $86 \%)$ voiced the opinion that augmented reality can support $\mathrm{ML}$ activities for non-experts.

The free-text comments provide further insights. Interactivity was repeatedly appreciated: "It is easier to memorize something when you experience it and this app is the closest you can get to experiencing something abstract with numbers and data". Similarly, "animations and connecting physical space to learnings helps remembering the learned concepts better. Here you can play around and learn from mistakes". Moreover, subjects mentioned that the user experience was engaging due to the use of immersion: "motivating because tech is cool". Specifically, immersion was found to be beneficial for non-experts without knowledge of ML: "higher immersion makes it more learnable" as the experience is "more personal than on the computer".

\section{Conclusion and Future Work}

In this paper, we presented ALEEDSA: an interactive system for machine learning with augmented reality. The effectiveness of our proof-of-concept was demonstrated in an early user study on credit risk modeling, which found that the use of augmented reality for interactive machine learning was perceived to be beneficial, motivating, and innovative.

Potential use cases of ALEEDSA span various settings in organizations and educational practices. First, augmented reality for machine learning promises to improve communication with domain experts, as ML can be made accessible to users without a technical background. Second, augmented reality supports users in understanding how ML models function. This is highly relevant as non-experts often perceive machine learning as a "black-box", and yet transparency in machine learning is a prerequisite in many domain applications (e.g., healthcare). Third, augmented reality for $\mathrm{ML}$ appears highly beneficial for education as it provides an engaging, peer-based learning experience.

Our early user feedback also revealed directions for future work. According to user feedback, the weight should be reduced and the field-of-view widened. We plan to achieve these improvements in usability by upgrading ALEEDSA to HoloLens 2. In addition, we intend to conduct additional experiments in order to evaluate the user experience.

\section{Acknowledgements}

Financial support as part of an ETH Innovedum grant (project: "rETHinking data science education in management: Collaborative learning in business analytics") is gratefully acknowledged. The authors thank Eeva Tervahartiala for her support with the user study. Stefan Feuerriegel was supported by a Digital Lives grant (183149) from the Swiss National Science Foundation (SNSF). 


\section{REFERENCES}

[1] Ronald T Azuma. 1997. A survey of augmented reality. In Presence: Teleoperators and Virtual Environments. Vol. 6. Cambridge, MA.

[2] Przemyslaw Biecek. 2018. Dalex: Explainers for complex predictive models in R. Journal of Machine Learning Research 19, 84 (2018).

[3] Mark Billinghurst, Adrian Clark, and Gun Lee. 2014. A survey of augmented reality. Foundations and Trends in Human-Computer Interaction 8, 2-3 (2014), 73-272.

[4] Long Chen, Thomas Day, Wen Tang, and Nigel W. John. 2017. Recent developments and future challenges in medical mixed reality. In IEEE International Symposium on Mixed and Augmented Reality (ISMAR)

[5] Finale Doshi-Velez and Been Kim. 2017. Towards a rigorous science of interpretable machine learning. arXiv:1702.08608 (2017).

[6] Yuichiro Fujimoto, Goshiro Yamamoto, Jun Miyazaki, and Hirokazu Kato. 2012. Relation between location of information displayed by augmented reality and user's memorization. In $A H$ '12: Proceedings of the 3rd Augmented Human International Conference.

[7] Sebastian Gehrmann, Hendrik Strobelt, Robert Kruger, Hanspeter Pfister, and Alexander M. Rush. 2019. Visual interaction with deep learning models through collaborative semantic inference. IEEE Transactions on Visualization and Computer Graphics 26, 1 (2019), 884-894.

[8] Riccardo Guidotti, Anna Monreale, Salvatore Ruggieri, Franco Turini, Dino Pedreschi, and Fosca Giannotti.
2018. A survey of methods for explaining black box models. ACM Computing Surveys (CSUR) 51, 5 (2018).

[9] Sebastian Günther, Florian Müller, Martin Schmitz, Jan Riemann, Niloofar Dezfuli, Markus Funk, Dominik Schön, and Max Mühlhäuser. 2018. CheckMate: Exploring a tangible augmented reality interface for remote interaction. In Conference on Human Factors in Computing Systems (CHI).

[10] Zachary C. Lipton. 2018. The mythos of model interpretability. Communications of ACM 61, 10 (2018), 35-43.

[11] Scott M Lundberg, Paul G Allen, and Su-In Lee. 2017. A Unified Approach to Interpreting Model Predictions. In Advances in Neural Information Processing (NIPS).

[12] Tom M. Mitchell. 1997. Machine Learning. McGraw-Hill, New York, NY.

[13] Sergio Orts-Escolano, Christoph Rhemann, Sean Fanello, Wayne Chang, Adarsh Kowdle, Yury Degtyarev, David Kim, Philip L Davidson, Sameh Khamis, Mingsong Dou, and et al. 2016. Holoportation: Virtual 3D teleportation in real-time. In Annual Symposium on User Interface Software and Technology (UIST). New York, NY, USA.

[14] Marco Tulio Ribeiro, Sameer Singh, and Carlos Guestrin. 2016. "Why should i trust you?" Explaining the predictions of any classifier. In ACM International Conference on Knowledge Discovery and Data Mining (KDD). 
[15] Marc Ericson C. Santos, Arno in Wolde Lübke, Takafumi Taketomi, Goshiro Yamamoto, Ma.

Mercedes T. Rodrigo, Christian Sandor, and Hirokazu Kato. 2016. Augmented reality as multimedia: The case for situated vocabulary learning. Research and Practice in Technology Enhanced Learning 11, 1 (2016), Article 4

[16] Ben Screck, Max Kanter, Veeramachaneni Kalyan, Vohra Sanjeev, and Prasad Rajendra. 2018. Getting value from machine learning isn't about fancier algorithms: It's about making it easier to use. Harvard Business Review (2018).
[17] Marcus Specht, Stefaan Ternier, and Wolfgang Greller. 2011. Mobile augmented reality for learning: A case study. (2011).

[18] Anna Syberfeldt, Oscar Danielsson, and Patrik Gustavsson. 2017. Augmented reality smart glasses in the smart factory: Product evaluation guidelines and review of available products. In IEEE Access, Vol. 5. 9118-9130.

[19] Ming Yin, Jennifer Wortman Vaughan, and Hanna Wallach. 2019. Understanding the effect of accuracy on trust in machine learning models. In Conference on Human Factors in Computing Systems (CHI). 